\title{
Negative impact of the COVID-19 state of emergency on breast cancer screening participation in Japan
}

\author{
Yasuhiro Toyoda $^{1,2}\left(\right.$ Cota Katanoda ${ }^{3} \cdot$ Kanako Ishii $^{4} \cdot$ Hitoshi Yamamoto $^{1} \cdot$ Takahiro Tabuchi $^{2}$
}

Received: 18 February 2021 / Accepted: 5 July 2021 / Published online: 9 July 2021

(c) The Japanese Breast Cancer Society 2021

\begin{abstract}
Background In response to the Coronavirus-19 (COVID-19) pandemic, the Japanese government declared a state of emergency in Saitama, Chiba, Tokyo, Kanagawa, Osaka, Hyogo and Fukuoka prefectures on April 7, 2020; this was extended to the remaining prefectures on April 16, 2020. The state of emergency was lifted on May 25, 2020. Although it was known that breast cancer screening was postponed or canceled during this period, the actual extent of postponement or cancellation has not been clarified.

Methods We investigated postponement or cancellation of breast cancer screening between April and May 2020 using a cross-sectional, web-based, self-reported questionnaire survey. In addition, we examined the association between socioeconomic and health-related factors and postponement or cancellation by multivariable log-binominal regression.

Results Among 1874 women aged 30-79 years who had scheduled breast cancer screening during the study period, 493 women (26.3\%) postponed or canceled screening. While women aged 30-39 years and 70-79 years postponed or canceled less frequently than women aged $40-49$ years (prevalence ratio $=0.62$ and 0.56 , respectively), there was no significant difference between age groups in the women aged 40-69 years. Postponement or cancellation was more frequent in five prefectures, where the state of emergency was declared early (prevalence ratio=1.25). Employment status, annual household income, family structure, academic background, smoking status, and fear of COVID-19 were not associated with postponement or cancellation.

Conclusion Although care should be taken with the interpretation of these findings due to possible biases, they suggest that the postponement or cancellation of breast cancer screening might be due more to facility suspension than to individual factors. It is necessary to explore the ideal way of encouraging breast cancer screening uptake, in an environment of coexistence with COVID-19.
\end{abstract}

Keywords Breast cancer · Breast cancer screening · COVID-19

Yasuhiro Toyoda

ytoyoda@osaka.med.or.jp

1 Department of Breast and Thyroid Surgery, Minoh City Hospital, 7-1, Kayano 5-chome, Minoh-shi, Osaka 562-0014, Japan

2 Cancer Control Center, Osaka International Cancer Institute, 1-69, Otemae 3-chome, Chuo-ku, Osaka-shi, Osaka 541-8567, Japan

3 Division of Cancer Statistics Integration, Center for Cancer Control and Information Services, National Cancer Center, 5-1-1 Tsukiji Chuo-ku, Tokyo 104-0045, Japan

4 Public Health, Department of Social Medicine, Osaka University Graduate School of Medicine, 2-2 Yamadaoka, Suita, Osaka 565-0871, Japan

\author{
Abbreviations \\ COVID-19 Coronavirus disease 2019 \\ FCV-19S Fear of Coronavirus-19 Scale \\ CSLC Comprehensive Survey of Living Conditions \\ JCA Japan Cancer Association
}

\section{Introduction}

Coronavirus disease 2019 (COVID-19) has spread globally, since it was confirmed in Wuhan, China in December 2019. In Japan, it spread rapidly following detection of the first case on January 15, 2020 [1-3]. In response to the COVID19 pandemic, the Japanese government declared a state of emergency in Saitama, Chiba, Tokyo, Kanagawa, Osaka, Hyogo and Fukuoka prefectures on April 7, 2020; this was 
extended to the remaining prefectures on April 16, 2020. As a result, restrictions were imposed on the Japanese people, such as school closures, suspension of public and private facilities, and the introduction of teleworking. The state of emergency was lifted on May 25, $2020[4,5]$.

There was also an impact on the clinical practice of breast cancer. For example, some medical facilities postponed scheduled surgery [2]. In addition, just as cancer screening was postponed or canceled worldwide due to the pandemic [6], so breast cancer screening was postponed or canceled during the state of emergency in Japan [7].

The Ministry of Health, Labor and Welfare recommends population-based breast cancer screening using biennial mammography for women aged 40 years or more. Furthermore, opportunistic breast cancer screening using mammography and/or ultrasonography has spread in Japan. The Comprehensive Survey of Living Conditions (CSLC: a nationwide population-based cross-sectional survey) found that in 2019, the breast cancer screening rate, including both population-based and opportunistic screening, among women aged 40-69 years was $48.9 \%$ [8]. This level decreased in 2020; however, as the actual extent of postponement or cancellation of breast cancer screening due to the COVID-19 pandemic in Japan has not been clarified, the purpose of the present study was to examine the impact of COVID-19 on breast cancer screening postponement or cancellation.

\section{Materials and methods}

\section{Study setting}

To investigate the social and health situation in relation to the pandemic in Japan, the Japan COVID-19 and Society Internet Survey (JACSIS), a cross-sectional, web-based, selfreported questionnaire survey was conducted from August to September 2020. We recruited participants from an internet survey agency (Rakuten Insight, Inc., Tokyo, Japan), by a random sampling method using a computer algorithm; the sample was representative of the official Japanese demographic composition as of October 1, 2019 based on categories of age, sex, and place of residence (i.e., prefecture). Distribution of the questionnaires started on August 25, 2020, and was completed on September 30, 2020, when the target numbers for each sex, age, and place of residence were met. Finally, a total of 224,389 panelists, aged $15-79$ years, were sent an e-mail invitation to participate. The response rate was $12.5 \%(28,000 / 224,389)$. The questionnaires consisted of approximately 600 items.

Of the 28,000 participants $(14,000$ men and 14,000 women) primarily registered with the JACSIS database, 2,518 were excluded because of invalid responses, leaving
25,482 (12,673 men and 12,809 women). Invalid responses comprised: participants who did not choose the correct item in response to the attention check: 'Please select the second item from the bottom' $(N=1995)$; participants who answered 'yes' for all the following items: 'Are you currently drinking or using alcohol or drugs? Please answer for each of the following. (i.e., sleeping pills, anxiolytic agents, legal opioids, illegal opioids, cannabis, cocaine, heroin, and stimulant drug)'; or 'Do you currently have a chronic illness? (16 items: i.e., diabetes, asthma, stroke, ischemic heart disease, cancer, mental disease etc.)' $(N=563)$.

\section{Statistical analyses}

Figure 1 shows the study profile. As mentioned above, 12,809 women registered in the JACSIS, and among those, 10,514 were aged $30-79$ years. We further excluded 8640 women who were not scheduled to have breast cancer screening between April 2020 and May 2020. The remaining 1874 women were analyzed in the present study.

We investigated the proportion of postponement or cancellation of scheduled breast cancer screening during April and May 2020, which corresponded to the period of the COVID-19 state of emergency in Japan. In addition, we examined the relationship between postponement or cancellation of breast cancer screening and socioeconomic and health-related factors.

The objective variable was postponement or cancellation of breast cancer screening determined with a yes/no answer to the question 'Was your breast cancer screening canceled or postponed between April 2020 and May 2020?' As mentioned above, women who answered 'not applicable' for this question $(N=8640)$ were excluded from the analysis.

In this study, breast cancer screening included both population-based and opportunistic cancer screening. There are various modalities of breast cancer screening, but this study does not provide details and considers all modalities including mammography, ultrasonography and palpation.

We adopted age, residential region, employment, household annual income, family structure, academic background, smoking status and fear of COVID-19 as the explanatory variables. Age was categorized into 10-year intervals (30-39, 40-49, 50-59, 60-69 and 70-79 years). Residential regions were categorized into the prefectures for which the state of emergency was declared on April 7, 2020 and those for which it was not. Employment was categorized into regular-employee, non-regular employee, self-employed and not-employed. Household annual income was categorized into unknown, less than 4 million Japanese Yen (JPY), 4 million JPY or more but less than 8 million JPY, and 8 million JPY or more. 'Unknown' consisted of those who answered, 'I don't want to answer' and 'I don't know,' and did not include missing values. 
From Rakuten Insight, random sampled 224,389 panelists, aged 15-79 years, representative of the official Japanese demographic composition were recruited to the JACSIS.

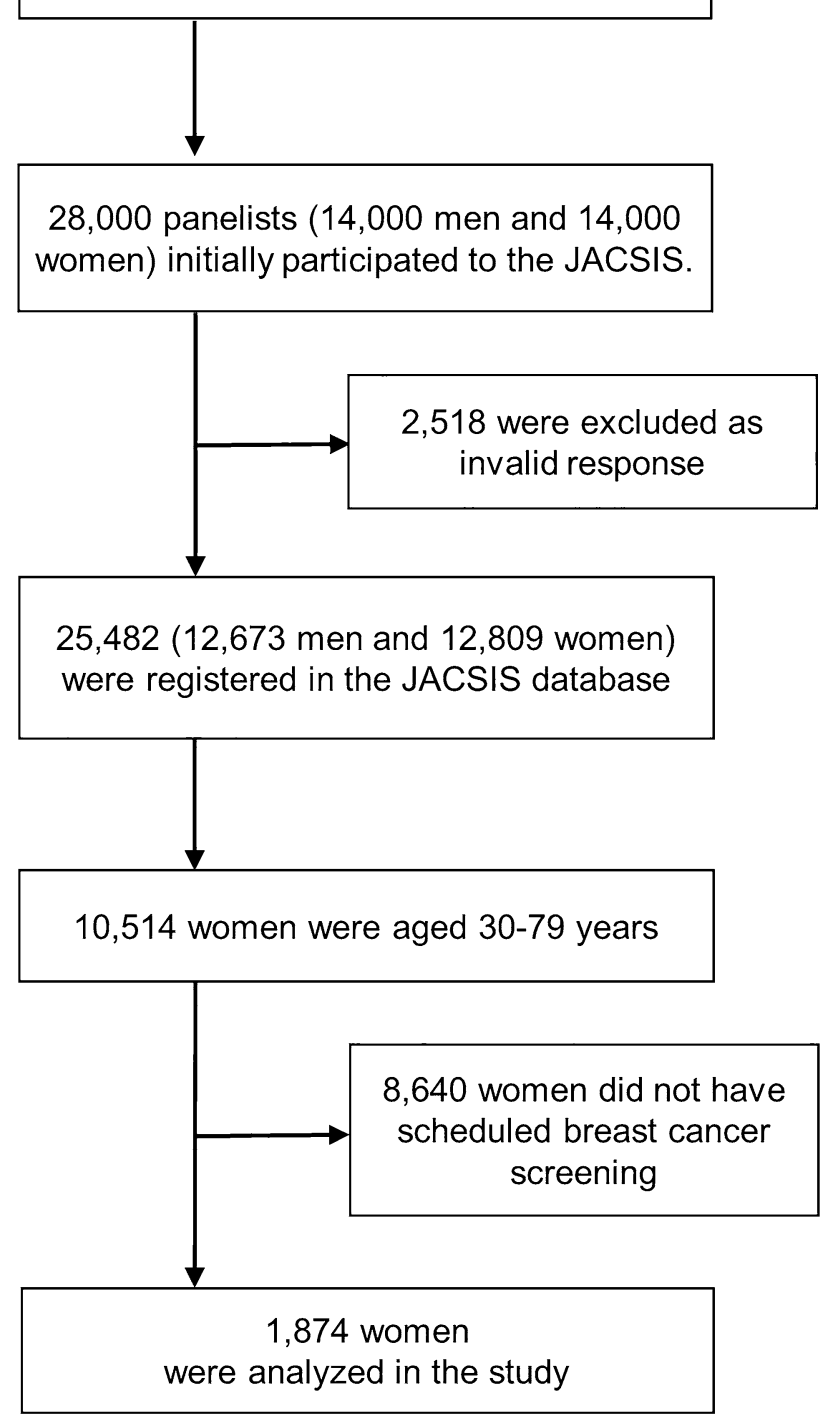

Fig. 1 Study profile

Family structure was categorized into living alone and not living alone. Academic background was categorized into 4-year university or graduate school and other. To estimate fear of COVID-19, we used the Japanese version of Fear of Coronavirus-19 Scale (FCV-19S) [9, 10]. The scale consisted of the following seven items:

1. I am most afraid of coronavirus-19.

2. It makes me uncomfortable to think about coronavirus-19.
3. My hands become clammy when I think about coronavirus-19.

4. I am afraid of losing my life because of coronavirus-19. 5 . When watching news and stories about coronavirus-19 on social media, I become nervous or anxious.

6 . I cannot sleep because I'm worrying about getting coronavirus-19.

7. My heart races or palpitates when I think about getting coronavirus-19.

Participants indicated their level of agreement with the statements using a five-item Likert-type scale. Answers were: 'strongly disagree,' 'disagree,' 'neither agree nor disagree,' 'agree,' and 'strongly agree'. The minimum score possible for each question was 1 , and the maximum was 5 . A total score was calculated by adding up each item score (ranging from 7 to 35). In the present study, FCV-19S was categorized into 7-15, 16-20, 21-25 and 26-35.

To calculate prevalence ratios and $95 \%$ confidence intervals of each socioeconomic and health factor, we used multivariable log-binomial regression models, because the outcome was more than $10 \%$ [11].

Probability values for statistical tests were two-tailed, and $P<0.05$ was considered statistically significant. All analyses were performed using SAS version 9.2 (SAS Institute, Cary, NC, USA).

\section{Ethical approval}

This study was reviewed and approved by the Research Ethics Committee of Osaka International Cancer Institute (approved on June 19, 2020; approval number 20084) and the National Cancer Center (approved on January 22, 2021; Protocol Number 2020-447). All participants provided webbased informed consent before responding to the online self-report questionnaire. Participants who completed the response were given points that could be used for online shopping and cashless shopping.

\section{Results}

Table 1 shows the number and proportion of postponements or cancellations and the multivariable-adjusted prevalence ratios. Among 1874 women who had scheduled breast cancer screening, 493 women $(26.3 \%)$ postponed or canceled their screening in April and May 2020.

While the frequencies of postponement or cancellation of breast cancer screening for women aged 30-39 years and $70-79$ years were lower than those for women $40-49$ years (prevalence ratio $=0.62$ and 0.56 , respectively), those for 50-59 years and 60-69 years were not statistically different from women aged $40-49$ years. 
Table 1 Multivariableadjusted prevalence ratios for postponement or cancellation of breast cancer screening

No. of postponements or cancella- $\quad(\%) \quad$ PR (95\% CI) tions of breast cancer screening

\begin{tabular}{|c|c|c|c|}
\hline \multicolumn{4}{|l|}{ Age, years } \\
\hline $30-39$ & $55 / 263$ & $(20.9)$ & $0.62(0.47-0.81)^{*}$ \\
\hline $40-49$ & $144 / 440$ & $(32.7)$ & 1 \\
\hline $50-59$ & $102 / 378$ & $(27.0)$ & $0.84(0.68-1.04)$ \\
\hline $60-69$ & $122 / 421$ & $(29.0)$ & $0.88(0.71-1.09)$ \\
\hline $70-79$ & $70 / 372$ & $(18.8)$ & $0.56(0.42-0.74)^{*}$ \\
\hline \multicolumn{4}{|c|}{ Residential region under state of emergency on April 7, 2020} \\
\hline Yes & $236 / 803$ & $(29.4)$ & $1.25(1.08-1.46)^{*}$ \\
\hline No & $257 / 1,071$ & $(24.0)$ & 1 \\
\hline \multicolumn{4}{|l|}{ Employment } \\
\hline Not-employed & $239 / 931$ & $(25.7)$ & 1 \\
\hline Self-employed & $27 / 102$ & $(26.5)$ & $0.94(0.67-1.33)$ \\
\hline Non-regular employee & $125 / 481$ & $(26.0)$ & $0.91(0.75-1.11)$ \\
\hline Regular employee & $102 / 360$ & $(28.3)$ & $0.98(0.78-1.22)$ \\
\hline \multicolumn{4}{|l|}{ Household annual income, JPY } \\
\hline$<4$ million & $142 / 552$ & $(25.7)$ & 1 \\
\hline$>=4$ million,$<8$ million & $146 / 571$ & $(25.6)$ & $0.93(0.75-1.14)$ \\
\hline$>=8$ million & $88 / 311$ & $(28.3)$ & $0.94(0.73-1.20)$ \\
\hline Unknown & $117 / 440$ & $(26.6)$ & $0.96(0.77-1.19)$ \\
\hline \multicolumn{4}{|l|}{ Family structure } \\
\hline Living alone & $62 / 254$ & $(24.4)$ & 1 \\
\hline Not living alone & $431 / 1,620$ & $(26.6)$ & $1.08(0.85-1.37)$ \\
\hline \multicolumn{4}{|l|}{ Academic background } \\
\hline 4-year university or graduate school & $168 / 585$ & $(28.7)$ & $1.09(0.92-1.28)$ \\
\hline Other & $325 / 1,289$ & $(25.2)$ & 1 \\
\hline \multicolumn{4}{|l|}{ Smoking status } \\
\hline Non smoker & $322 / 1,284$ & $(25.1)$ & 1 \\
\hline Ex-smoker & $112 / 373$ & $(30.0)$ & $1.16(0.97-1.39)$ \\
\hline Current smoker & $59 / 217$ & $(27.2)$ & $1.02(0.80-1.29)$ \\
\hline \multicolumn{4}{|l|}{ FCV-19S } \\
\hline $7-15$ & $103 / 391$ & $(26.3)$ & 1 \\
\hline $16-20$ & $164 / 581$ & $(28.2)$ & $1.08(0.88-1.33)$ \\
\hline $21-25$ & $142 / 570$ & $(24.9)$ & $0.99(0.79-1.24)$ \\
\hline $26-35$ & $84 / 332$ & $(25.3)$ & $1.02(0.79-1.31)$ \\
\hline Total & $493 / 1,874$ & (26.3) & \\
\hline
\end{tabular}

$P$ value for log-binomial regression analysis

$P R$ prevalence ratio, $C I$ confidence interval, $F C V$-19S Fear of Coronavirus-19 Scale, JPY Japanese yen $* P<0.05$

Participants who lived in prefectures for which a state of emergency was declared on April 7, 2020 canceled or postponed significantly more frequently (prevalence ratio $=1.25$ ). The other explanatory variables-employment, household annual income, family structure, academic background, smoking status and FCV-19S were not statistically significant.

\section{Discussion}

In the present study, $26.3 \%$ of breast cancer screening appointments were postponed or canceled during the 2 months of the state of emergency. However, the Japan Cancer Association (JCA) reported the number of 
population-based stomach, colorectal, lung, breast and cervical cancer screenings in May 2020 reduced to approximately 10\% of that in May 2019 [12]. The simple assumption from this report is that based on the previous year's figures, approximately $90 \%$ of population-based cancer screenings were not performed. There are several possible reasons for this substantial difference between the present study and the JCA report.

First, while the JCA report focused on the decrease in the number of screenings performed, the subject of present study was the postponement or cancellation of scheduled screening. Since the decrease in scheduled screening was not covered in the JCA report, a direct comparison with the present study was not possible. However, there are biases in the subjects of the present study and they may not necessarily represent the general population. We defined having scheduled breast cancer screening as those participants who answered 'yes' or 'no' to the question: 'Was your breast cancer screening canceled or postponed between April 2020 and May 2020?'. Participants who answered 'not applicable' were assumed to have had no scheduled screening, and were excluded from the analyses. However, recognition and interpretation of 'scheduled screening' might vary between participants. Some participants may have interpreted 'scheduled screening' as having an appointment, others only as having the intention to attend screening. The variation might lead to an over-estimation of scheduled screening. In addition, some participants might have answered 'no' even though they had no screening scheduled. This could cause an underestimation of postponement or cancellation. Second, the present study includes population-based cancer screening but also opportunistic cancer screening. According to CSLC in 2016, the proportion of opportunistic screening compared to total breast cancer screening for women aged 30-79 years was 57\% [personal communication: Ishii K, April 16, 2021]. There might be differences between population-based and opportunistic screening in terms of decreased appointment numbers. Thirdly, some participants might confuse breast cancer screening and clinical follow-up of breast findings.

It was assumed that two main factors relate to postponement or cancellation of cancer screening: (1) the behavior of the screenee and (2) the decision of the screening facility. Some screenees might have spontaneously postponed or canceled their screening appointment because of the COVID-19 pandemic. On the other hand, some screening facilities suspended and stopped screening and health checkups after the state of emergency was declared [7]. Several characteristics which promote breast cancer screening participation have been reported in previous pre-COVID-19 studies: $40 \mathrm{~s}$ age group, high final academic achievement, regular-employee, high income, not living alone and nonsmoking [13-17]. It seemed that women with these characteristics are highly conscious of their health. It was thus likely that when high health awareness also means high COVID-19 awareness, women with these characteristics would more frequently postpone or cancel breast cancer screening. However, in the present study, these socioeconomic factors were not associated with postponement or cancellation of breast cancer screening.

Regarding age group, Japanese women aged 40-49 years more frequently participate in breast cancer screening than women aged 50-59 or 60-69 years [16]. In the present study, although women aged $70-79$ years and $30-39$ years postponed or canceled breast cancer screening less frequently than women aged 40-49 years, age group was not associated with postponement or cancellation of breast cancer screening in the majority of screenees (aged 40-69 years).

Several factors might explain why women aged 30-39 years and 70-79 years postponed or canceled less frequently. In general, women aged 30-39 years were outside the breast cancer screening program age. However, it is possible that women aged 30-39 years analyzed in the present study made appointments for screening themselves. In other words, they were highly conscious of breast cancer risk and would not postpone or cancel an appointment for screening. On the other hand, it is characteristic of elderly Japanese women to avoid involvement in medical decisions and follow the instructions of their health care professional [18]. Women aged 70-79 years in the present study might, therefore, be unlikely to postpone or cancel an appointment for breast cancer screening.

FCV-19S, which was developed to estimate the fear of COVID-19, was also not associated with postponement or cancellation of breast cancer screening.

While postponement or cancellation were not associated with these individual factors, except for age group, they occurred more frequently in the prefectures, where the state of emergency was declared early: Saitama, Chiba, Tokyo, Kanagawa, Osaka, Hyogo and Fukuoka. These findings suggest that the postponement or cancellation of breast cancer screening might more often be due to facility suspension than to individual factors.

The present study had some limitations. First, despite random sampling, there is selection bias, because the survey uses self-reported questionnaires. In addition, as mentioned above, some participants might be confused by or misunderstand the question. There are biases among the subjects of the present study and they may not necessarily represent the general population.

Second, participants responded in August-September 2020, but were asked to remember April-May 2020, which may lead to recall bias. Finally, given the cross-sectional nature of the study, we could not identify the causal relationship between postponement or cancellation of breast cancer screening and the explanatory variables. However, explanatory variables such as residential region would not 
significantly change over time. Furthermore, a follow-up study of the JACSIS is now ongoing.

In conclusion, although care should be taken in the interpretation of these findings due to biases, in a cross-sectional, web-based, self-reported questionnaire survey, approximately $26 \%$ of breast cancer screening was postponed or canceled due to the COVID-19 state of emergency. The postponement or cancellation of breast cancer screening might more often be due to facility suspension than to individual factors. The interruption of breast cancer screening due to COVID-19 might cause an increase in advanced breast cancer incidence and breast cancer mortality [19-21]. It is, therefore, necessary to explore the ideal way of maintaining breast cancer screening uptake in an environment of coexistence with COVID-19.

Acknowledgements This study was funded by the Japan Society for the Promotion of Science (JSPS) KAKENHI Grants (grant number 18H03062 and 21H04856, Dr. Takahiro Tabuchi; 17H03589, Dr. Kota Katanoda; 19K10671, Dr. Keisuke Kuwahara; 19K19439, Dr. Kanami Tsuno; 19K10446, Dr. Kenji Takeuchi; 18H03107, Dr. Hiroshi Murayama), Research Support Program to Apply the Wisdom of the University to tackle COVID-19-Related Emergency Problems, University of Tsukuba (Dr. Ai Hori), Health Labour Sciences Research Grants (grant number 19FA1005, Dr. Isao Muraki; 19FA1012, Prof. Naoki Kondo), and the Japan Agency for Medical Research and Development (AMED; grant number 2033648, Prof. Takeo Fujiwara). We thank Dr. J. Mortimer for her English language editing.

\section{Declarations}

Conflict of interest Kota Katanoda received a JMWH Bayer Grant (funded by Bayer) from the Japan Society for Menopause and Women's Health. This financial relationship is outside the present study and did not affect any part of the work. The other authors have no conflicts of interest.

\section{References}

1. Kuniya T. Evaluation of the effect of the state of emergency for the first wave of COVID-19 in Japan. Infect Dis Model. 2020;5:580-7.

2. Watanabe M. The COVID-19 pandemic in Japan. Surg Today. 2020;50:787-93.

3. Furuse Y, Ko YK, Saito M, Shobugawa Y, Jindai K, Saito T, et al. Epidemiology of COVID-19 outbreak in Japan, from JanuaryMarch 2020. Jpn J Infect Dis. 2020;73:391-3.

4. Looi MK. Covid-19: Japan declares state of emergency as Tokyo cases soar. BMJ. 2020;369: m1447. https://doi.org/10.1136/bmj. $\mathrm{m} 1447$

5. Looi MK. Covid-19: Japan prepares to extend state of emergency nationwide as "untraceable" cases soar. BMJ. 2020;369: m1543. https://doi.org/10.1136/bmj.m1543.

6. Mitchell EP. Declines in cancer screening during COVID-19 pandemic. J Natl Med Assoc. 2020;112:563-4.
7. Japan Society of Ningen Dock. Questionnaire for COVID-19 (2020). https://www.ningen-dock.jp/wp/wp-ontent/uploads/2020/ 03/46b326d938d5e1f93926958d45af5046.pdf. Accessed 1 Feb 2021 (in Japanese).

8. Statistics of Japan. National Statistics Center. Cancer Screening Rate (2020). https://www.e-stat.go.jp/stat-search/files?page= $1 \&$ layout $=$ datalist $\&$ touke $=00450061 \&$ tstat $=000001141126 \&$ cycle $=7 \&$ tclass $1=000001141142 \&$ tclass $2=000001142126 \&$ tclas s3val $=0$. Accessed 19 Apr 2021 (in Japanese)

9. Ahorsu DK, Lin CY, Imani V, Saffari M, Griffiths MD, Pakpour AH. The Fear of COVID-19 Scale: development and initial validation. Int J Ment Health Addict. 2020;27:1-9.

10. Wakashima K, Asai K, Kobayashi D, Koiwa K, Kamoshida S, Sakuraba M. The Japanese version of the Fear of COVID-19 scale: reliability, validity, and relation to coping behavior. PLoS ONE. 2020;15(11):e0241958.

11. McNutt LA, Wu C, Xue X, Hafner JP. Estimating the relative risk in cohort studies and clinical trials of common outcomes. Am J Epidemiol. 2003;157:940-3.

12. Japan Cancer Association. Changes in the number of cancer screening examinees (2021). https://www.mhlw.go.jp/content/ 10901000/000751177.pdf. Accesesd 13 Apr 2021 (in Japanese)

13. Tsunemastu M, Kawasaki H, Masuoka Y, Kakehashi M. Factors affecting breast cancer screening behavior in japan-assessment using the health belief model and conjoint analysis. Asian Pac J Cancer Prev. 2013;14:6041-8.

14. Kim J, Jang SN. Socioeconomic disparities in breast cancer screening among US women: trends from 2000 to 2005. J Prev Med Public Health. 2008;41:186-94.

15. Watanabe R. An analysis of participation in cancer screening in japan. Iryo to Shakai. 2003;13:113-32 (in Japanese).

16. Clark MA, Rakoeski W, Ehrich B. Breast and cervical cancer screening: association with personal, spouse's, and combined smoking status. Cancer Epidemol Biomarkers Prev. 2000;9:513-6.

17. Ohara K, Saeki K, Nezu T, Obayash K, Tomioka K, Okamoto N, et al. Examination of behavioral determinants of cancer screening. Kosei no Shihyo. 2014;61:13-20 (in Japanese).

18. Ohki M. Measurement of decision-making with respect to medical practices by Japanese. Ningen Kagaku Kenkyu (Bulletin of Human Science). 2005;27:83-92 (in Japanese).

19. Maringe C, Spicer J, Morris M, Purushotham A, Nolte E, Sullivan $\mathrm{R}$, et al. The impact of episodic screening interruption: COVID-19 and population-based cancer screening in Canada. J Med Screen. 2020;28:0969141320974711. https://doi.org/10.1177/0969141320 974711 (Online ahead of print).

20. Vanni G, Pellicciaro M, Materazzo M, Bruno V, Oldani C, Pistolese CA, et al. Lockdown of breast cancer screening for COVID19: possible scenario. In Vivo. 2020;34:3047-53.

21. Maringe C, Spicer J, Morris M, Purushotham A, Nolte E, Sullivan $\mathrm{R}$, et al. The impact of the COVID-19 pandemic on cancer deaths due to delays in diagnosis in England, UK: a national, populationbased, modelling study. Lancet Oncol. 2020;21:1023-34.

Publisher's Note Springer Nature remains neutral with regard to jurisdictional claims in published maps and institutional affiliations. 